\title{
Pyrexia in hospitalised spinal cord injury patients
}

\author{
P S S Beraldo MD, E G C Neves MD, C M F Alves MD, P Khan MD, A C M Cirilo \\ MD, M R B Alencar MD \\ Hospital for Medicine of the Locomotor System (HMLS)/SARAH, Brasília/DF, Brazil.
}

To discover the frequency of pyrexia in spinal cord injury (SCI) patients in the Hospital for Medicine of the Locomotor System (HMLS/SARAH), a review study was undertaken. For a period of 3 months, all new episodes of fever were recorded. During the study 221.3 patients/month were observed. The age ranged from 14 to 60 years and time of lesion from 1 to 6,264 days: 85 patients were paraplegia or paraparetic, and 44 tetraplegic or tetraparetic. At the beginning of the study $10.8 \%$ of the patients had fever. During follow up, we observed a rate of 33.9 new incidences per 100 patients/month. In $77.3 \%$ only one cause was identified, and in $8 \%$ no cause could be defined. The commonest causes were urinary tract infection (UTI) $(44.3 \%)$, and soft tissue infections $(11.4 \%)$. Frequent causes of fever in the acute stage other than UTI were complications related to the initial trauma and thromboembolic disease $(p<0.05)$. These results demonstrate the high incidence of fever in patients with SCI, in addition to providing a useful approach for diagnosis and management.

Keywords: fever; pyrexia; spinal cord injury; morbidity; urinary tract infection.

\section{Introduction}

Various causes including infections are responsible for the significant morbidity and mortality in patients with SCI. ${ }^{1.2}$ Fever is frequently the first and may be the only manifestation of a complication, and can provide a diagnostic challenge. Paradoxically there are few studies dedicated to the quantification and to the diagnosis of the causes of fever in such patients, such as is given in this present investigation.

\section{Patients and methods}

The study consisted of a first phase where a detailed survey was carried out of all hospitalised patients with SCI in the Hospital for Medicine of the Locomotor System (HMLS/SARAH) on 5 July 1989. Besides the clinical features, a record was made of all patients with more than two episodes of fever (axillary temperature $\geqslant 38^{\circ} \mathrm{C}$ ) for 24 hours before and whilst undergoing treat- ment for a clinical condition associated with fever. All episodes of fever were also recorded in charts during the hospitalisation. From this date and during the following 3 months, all new episodes of fever (two or more episodes over a 24 hour period) were recorded.

The following criteria for the aetiology of the fever were established:

Urinary tract infection (UTI). Patients with indwelling bladder catheters and pyuria, with a white blood cell level of more than 20 per high power field (WBC/HPF). or urine culture with bacterial colony counts of greater than 100,000 colonies/ml, and also the finding of urine turbidity and a dense precipitate. In patients who did not have indwelling catheters or were undergoing intermittent catheterisation, the presence of pyuria greater than $10 \mathrm{WBC} / \mathrm{HPF}$ and a urine culture with bacterial colony counts greater than 100,000 colonies $/ \mathrm{ml}$ were included. In these patients the blood counts should be compatible with urinary 
infection, with no evidence of genital herpes simplex infection or vaginitis. Orchiditis or epididymitis was diagnosed by the presence of enlargement of the organs, associated with a compatible echography.

Deep-vein thrombosis. Swelling of the limb with an increase of calf and thigh circumference, and if present the finding of a positive Homans sign. Abnormalities on phlebography, echo-doppler and/or compatible scintillography, confirm the diagnosis.

Bacterial pneumonia. Cough, yellow sputum, chest xrays showing consolidation and a positive bacterial sputum culture. Pulmonary contusion and pleural effusion secondary to the trauma of the thoracic region were considered to be the cause of fever when they were in keeping with the patient's history, physical examination, and chest xrays, and in the absence of the other criteria for bacterial pneumonia.

Infected decubitus ulcer (DU). Presence of pus, necrotic tissue, with or without an abscess. 'Immediate postsurgical' fever was classified as the febrile episode restricted to the period of 48 hours post surgery with a spontaneous resolution in patients submitted to surgical treatment for DU. Febrile episodes after this period associated with the surgical site (suppuration, dehiscence) were considered as late complications and classified as 'postsurgical repair'.

Osteomyelitis. Xrays and a bone scan with compatible abnormalities, positive bacterial culture of the presence of pus cells from biopsy of the suspected material.

Ear, nose, throat infections. Throat pain, coryza, inflamed oropharynx and submandibular adenopathy, possibly leading to a dry cough but with normal xrays of the chest.

Sepsis. At least three positive blood cultures. Clinical symptoms of tachycardia, tachypnea, manifestations of inadequate perfusion or organic abnormalities - alterations of behaviour, hypoxia, metabolic acidosis and/or oliguria.

Pulmonary embolism. Positive clinical evidence or a history of a previous deepvein thrombosis. Arterial blood gases with hypoxia, abnormal LDH, modification of the electrocardiogram, associated altera- tions in chest xrays. A lung scan was used to confirm diagnosis, particularly if areas of decreased perfusion were present and chest xrays demonstrated no parenchymal disease.

Drug induced fever. Exclusion of the other causes associated with the disappearance of the fever within the maximum of 48 hours after the withdrawal of the suspected drug. Drug induced fever was considered if on readministration of the drug, the fever recurred after a short period. Patients who had fever without a precise diagnosis, even after routine laboratory tests, were investigated more thoroughly with additional tests.

In the analysis of the results, we used a software microcomputer EPI INFO (version 5). For statistical analysis, chi square and Fisher exact test were used when indicated, with $5 \%$ as the limit for significance.

\section{Results}

On the day of the initial investigation, there were 74 hospitalised patients. During the 3 months of observation, 48 patients were discharged and 58 were admitted. Considering the number of patients in the study during 3 months, there was a total of 221.3 patients/month.

The age of the population in the study ranged from 14 to 60 years. There was a predomination of males, 98 men, with a male:female ratio of $3.1: 1.0$. The duration of the lesion at the time of the study ranged from 1 to 6264 days; $54.6 \%$ of patients were admitted within the first 90 days of lesion. Analysing the level and degree of lesion, 85 patients presented thoracolumbar lesions (65 complete) and 44 cervical lesions (10 complete) and one patient had no presenting neurological deficit. Traffic accidents $(48.5 \%)$, firearm $(24.6 \%)$ and fall/diving $(22.5 \%)$ were the principal causes of lesions.

Analysing those patients who had fever or were receiving specific treatment on the first day of the investigation, we found 8 patients, a point prevalence of $10.8 \%$ $(8 / 74)$. Seven of the 8 patients with fever had only one cause for the febrile picture: 4 urinary infection, 1 deep-vein thrombosis, 
1 bacterial pneumonia and 1 with an abscess in the ischial region where the patient had a DU. Only one patient had three causes: a DU in the sacral region, urinary infection and deep-vein thrombosis. Furthermore, 36 patients $(48.7 \%)$ had a previous record in their charts of a febrile picture before the day of the investigation and during the same hospitalisation.

During the follow up 75 new febrile pictures were recorded in 56 patients, 33.9 new pictures per 100 patients/month. In 58 patients $(77.3 \%)$ only one cause for the febrile picture was identified, in 9 patients $(12.0 \%)$ two causes, in 2 patients $(2.7 \%)$ three or more causes and in 6 patients $(8 \%)$ no identifiable cause for the fever could be discovered.

The most frequent causes were urinary infection $(44.3 \%)$, soft tissue infections (including decubitus ulcer and abscess)
$11.4 \%$, postreconstructive surgery for decubitus ulcers $(9.1 \%)$ and uncomplicated upper respiratory tract infections $(8 \%)$. Other causes of fever were osteomyelitis $(2.3 \%)$ and bacterial pneumonia $(3.4 \%)$ (Table I).

No correlation between the percentage of patients with febrile disorders and the level of the lesion was observed $\left(x^{2}=0.05\right.$, $p>0.05)$. Analysing the incidence of fever from the time of lesion (that is 90 days), it was similar, only the causes being different. Urinary tract infection was the principal cause in a similar percentage, $48.8 \%$ and $42.4 \%$, respectively $\left(x^{2}=0.04, p>0.05\right)$, in both periods. Pulmonary complications (contusion and effusion) related to the initial trauma and deep-vein thrombosis/ pulmonary embolism (Fisher exact test, $p<0.05$ ) predominated in the acute stage (first 90 days), each with $11.6 \%$ of the causes, while none of these were observed

Table I Causes of fever exhibited by SCI patients during the 3 months follow up

\begin{tabular}{|c|c|c|c|c|c|c|}
\hline \multirow[t]{3}{*}{ Causes of fever } & \multicolumn{4}{|c|}{$\begin{array}{l}\text { Number of causes by } \\
\text { time of traumatic lesion }\end{array}$} & \multicolumn{2}{|r|}{ Total $(\%)$} \\
\hline & \multicolumn{2}{|c|}{$<90$ day } & \multicolumn{2}{|c|}{$>90$ days } & & \\
\hline & $N$ & $(\%)$ & $N$ & $(\%)$ & & \\
\hline Urinary tract infection & 21 & $(48.8)$ & 19 & $(42.2)$ & 39 & $(44.3)$ \\
\hline Soft tissue & 5 & $(11.6)$ & 13 & $(28.9)$ & 18 & $(20.5)$ \\
\hline decubitus ulcer (DU) & 1 & $(2.3)$ & 1 & $(2.2)$ & 2 & $(2.3)$ \\
\hline DU with contiguous abscess & 1 & $(2.3)^{\mathrm{c}}$ & 7 & $(15.6)$ & 8 & $(9.1)$ \\
\hline postsurgical repair & 1 & $(2.3)$ & 4 & $(8.9)$ & 5 & $(5.7)$ \\
\hline immediate postsurgical & 2 & $(4.6)$ & 1 & $(2.2)$ & 3 & $(3.4)$ \\
\hline Osteomyelitis & 1 & $(2.3)$ & 1 & $(2.2)$ & 2 & $(2.3)$ \\
\hline Respiratory tract & 9 & $(20.9)$ & 6 & $(13.3)$ & 15 & $(17.0)$ \\
\hline pneumonia & 2 & $(4.6)$ & 1 & $(2.2)$ & 3 & $(3.4)$ \\
\hline $\begin{array}{l}\text { pulmonary contusion and } \\
\text { effusion }{ }^{\mathrm{a}}\end{array}$ & 5 & $(11.6)^{t}$ & 0 & (0) & 5 & $(5.7)$ \\
\hline others $^{\mathrm{b}}$ & 2 & $(4.6)$ & 5 & (11.1) & 7 & $(8.0)$ \\
\hline Thromboembolic disease & 5 & $(11.6)^{\mathrm{t}}$ & 0 & (0) & 5 & $(5.7)$ \\
\hline pulmonary thromboembolism & 2 & $(4.6)$ & 0 & (0) & 2 & $(2.3)$ \\
\hline deep-vein thrombosis & 3 & $(6.9)$ & 0 & (0) & 3 & $(3.4)$ \\
\hline Miscellaneous $^{\mathrm{c}}$ & 1 & $(2.3)$ & 1 & $(2.2)$ & 2 & $(2.3)$ \\
\hline Unknown cause & 1 & $(2.3)$ & 5 & $(11.1)$ & 6 & $(6.8)$ \\
\hline Total of causes & 43 & $(100.0)$ & 45 & $(100.0)$ & 88 & $(100.0)$ \\
\hline Total of fever pictures & 32 & & 43 & & 75 & \\
\hline Total of patients ${ }^{d}$ & 25 & & 34 & & 62 & \\
\hline
\end{tabular}

" related to initial trauma. ${ }^{\mathrm{b}}$ sinusitis, viral respiratory infections, tonsillitis. c orchitis and varisela. d 5 patients were counted twice, since they presented fever in both periods after lesion. c $p=0.034$, between $<90$ and $>90$ days (Fisher exact test) ${ }^{\mathrm{f}} p=0.25$. 
later. After the acute period following the lesion, besides UTI, the causes were found to be probably related to soft tissue infections in $28.9 \%$, compared with $11.6 \%$ in the acute phase, although not significant statistically $\left(x^{2}=3.04, p>0.05\right)$, but with a significant percentage of decubitus ulcers with contiguous abscess in $15.6 \%$ compared with $2.3 \%$ in the acute phase (Fisher exact test, $p<0.05$ ). Unexplained fever was common in the acute stage, $11.1 \%$ and $2.3 \%$, respectively, but not significant (Fisher exact test, $p>0.05)$. No deaths were registered during the study related to a febrile disease.

At the time of the diagnosis, more than half $(22,56 \%)$ of the patients that developed urinary tract infection had indwelling bladder catheters and 8 were on intermittent catheterisation programmes. In $74.4 \%$ of the cases of UTI only one bacteria was isolated from culture, Escherichia coli predominating $(23.5 \%)$, then Klebsiella $s p$. $(19.6 \%)$ and Streptococcus faecalis $(11.8 \%)$.

\section{Discussion}

The one-year mortality rate for SCI patients with complete and incomplete paralysis is, respectively, $28 \%$ and $14 \% .^{3}$ The cost of medical and social assistance (USA) exceeds 7.4 billion dollars a year. ${ }^{+}$In Brazil this problem is not any less, with an estimated $8.6 \%$ patients admitted to orthopaedic and traumatology wards. ${ }^{5}$

The day to day ward experience with these patients has demonstrated that febrile episodes are frequent and their origins sometimes difficult to diagnose. Altered sensation and inability to control body temperature may contribute to this fact.$^{6}$ In this context, paradoxically few studies have apparently addressed the magnitude of the problem.

Pyrexia in the SCI population, found in this study, was very revealing. There was a point prevalence of $10.8 \%$, and it was observed that $49 \%$ of the patients presented during the period of hospitalisation when there was a record of at least one febrile episode in their temperature charts before the start of our study; an incidence of 34 new instances per 100 patients/months, approximately one new patient daily per 100 patients. We would emphasise that $73 \%$ of the all febrile episodes had infectious causes; within 3 months of lesion, noninfectious causes, thromboembolic disease and pulmonary complications related to the initial trauma, occurred in a similar percentage; $15 \%$ of the cases of all fevers had two or more causes, and in $8 \%$ of the cases there was no apparent cause based on the criteria being used. These results were confirmed in one of the few studies carried out on this topic, where high percentages were recorded by Sugarman et al ${ }^{1}$ In this study it was demonstrated that $67 \%$ of SCI patients in a specialised centre, with a median of 3 months after injury, had at least one febrile illness during the hospital stay; $81 \%$ were caused by infections, and in $11 \%$ the cause was not determined.

In all the studies UTI has been shown to be the most frequent complication in the SCI population, even with the use of the modern techniques of bladder management. ${ }^{7.8}$ More than $80 \%$ of the patients in this study were undergoing intermittent catheterisation; even so, UTI was responsible for $44 \%$ of the causes of fever. Sugarman et al found UTI in 39\% of his patients with or without fever.

DU is a frequent complication in SCI patients, particularly when these patients are not admitted immediately to a specialised centre. ${ }^{2}$ This is still one of the most frequent complications in Brazil, where there are few specialised centres and more than $50 \%$ of the hospitalised SCI patients had DU. ${ }^{5}$ In the present series, $60 \%$ of the patients had DU on admission, but none developed during hospitalisation. Direct and indirect (surgical procedures, osteomyelitis) conditions and complications related to soft tissue damage were responsible for $22.8 \%$ of the episodes of fever, mainly if the patients were within more than 90 days of lesion.

Thromboembolic disease is a leading cause of morbidity and mortality in patients with SCI. The incidence of deep-vein thrombosis in such patients ranges from $20 \%$ to $60 \%$ in reported series, depending on the type of hospital, time of lesion and diagnostic methodology used. ${ }^{9.10}$ This com- 
plication tends to occur within the first 3 months after initial injury; thereafter the incidence of deep-vein thrombosis appears to decline dramatically. ${ }^{11.12}$ Our results were compatible with those in the literature, corresponding to $11.6 \%$ of the causes of fever in the first 3 months of lesion. No case was observed after this period. We should emphasise that in our institution all patients receive prophylatic heparin routinely in the first 2 months after the lesion, in accord with recommendations in the literature. ${ }^{13}$ Deepvein thrombosis can also be present with fever as its only evidence; this fact should always be remembered as a differential diagnosis in SCI patients. ${ }^{14}$

As HMLS/SARAH is a rehabilitation institution, generally only clinically stable SCI patients are admitted for initial evaluation and rehabilitation. This could explain the absence or low incidence of certain potentially more serious causes of fever. The current literature has demonstrated a high incidence of other complications, particularly in high level SCI, frequently associated with fever, such as pancreatitis and upper gastrointestinal perforation, ${ }^{15}$ osteomyelitis and heterotopic bone formation, ${ }^{16.17}$ sepsis, pulmonary embolism and other pulmonary complications. ${ }^{18.19}$

The 8 patients who presented with fever without a precise diagnosis, all with a good and spontaneous resolution of the fever, presented with lesions between $\mathrm{C} 3$ and $\mathrm{T} 10$. We know that animals submitted to medullary lesions can behave as poikilotherms when exposed to ambient temperatures greater than $26.6{ }^{\circ} \mathrm{C} .^{20}$ In this series, none of the patients presented head trauma or associated factors affecting the central thermoregulation pathways. The mean temperature in Brasilia during the 3-month period of observation was $22^{\circ} \mathrm{C}$, ranging from 15 to $30^{\circ} \mathrm{C}$. Since self limited and short febrile episodes that lasted 24 hours or less were not evaluated, we cannot characterise the real problem of episodic fever in these patients. Sugarman ${ }^{21}$ and Sugarman et al proposed initially, and subsequently used, the term 'quadriplegia fever'. We suggest 'SCI fever' as a better term to express this condition, since paraplegics also exhibited fever without present adequate explanation in the study. This fact cannot be explained simply by the loss of thermoregulatory control and may be related to subclinical conditions, like benign thromboembolic disease, initial trauma, etc.

Regarding fever, we must remember Atkins and Bodel's ${ }^{22}$ statement: 'Fever is perhaps the most ancient and widely known hallmark of disease. For much of history the word fever has been used almost synonymously with disease .... These words are particularly true for the SCI population. The Latin proverb, Felix qui potuerit rerum causas cognoscere (Fortunate is he who is able to discern the causes of things), leaves us not happier but perhaps slightly wiser. The causes of things are not always simple to determine, nor are the results predictable, particularly concerning SCI patients.

\section{References}

1 Sugarman B, Brown D, Musher D (1982) Fever and infection in spinal cord injury patients. JAMA 248: 66-70.

2 Donovan WH, Carter RE, Bedbrook GM, Young JS, Griffiths ER (1984) Incidence of medical complications in spinal cord injury: patients in specialised, compared with non-specialised centres. Paraplegia 22: 282-290.

3 Esard L, Carmody A, Mannarino E, Ruge D (1978) Survival after spinal cord trauma. A life table analysis. Arch Neurol 35: 78-83.

4 Center for Disease Control (1988) Acute traumatic spinal cord injury surveillance - United States, 1987. JAMA 259: 3108 .

5 Campos Da Paz Jr A, Beraldo PSS, Almeida MCRR. Neves EGC. Alves CMF, Khan P (1992) Traumatic injury to the spinal cord. Prevalence in Brazilian hospitals. Paraplegia 30: 636-640.

6 Guttmann L, Silver J, Wyndham CH (1958) Thermoregulation in spinal man. J Physiol 142: 406-419.

7 Silver JR, Gibbon NOK (1968) Prognosis in tetraplegia. Br Med J 4: 79-83.

8 Dietz JM, Bertschy M, Gschaedler R. Dollfus P (1986) Reflections on the intensive care of 106 acute cervical spinal cord injury patients in the resuscitation unit of a general traumatology centre. Paraplegia 24: $343-349$. 
9 Todd JW, Frisbie JH, Rossier AB, Adams DF, Als AV, Armenia RJ et al (1976) Deep venous thrombosis in acute spinal cord injury: comparison of $\mathrm{I}_{125}$ fibrinogen leg scanning, impedance plethysmography and venography. Paraplegia 14: 50-55.

10 Branch BB, Moser KM, Cedar L, Minteer M, Convery R (1977) Venous thrombosis in acute spinal cord paralysis. J Trauma 17: 289-291.

11 El Marsri WS, Silver JR (1981) Prophylactic anticoagulant therapy in patients with spinal cord injury. Paraplegia 19: 334-342.

12 Watson N (1974) Anticoagulant therapy in treatment of venous thrombosis and pulmonary embolism in acute spinal cord injury. Paraplegia 12: 197-201.

13 Casas ER, Sanchez MP, Arias CR, Masip. JP (1976) Prophylaxis of venous thrombosis and pulmonary embolism in patients with traumatic spinal cord lesions. Paraplegia 14: 178-183.

14 Weingarden DS, Weingarden SI, Belen J (1987) Thromboembolic disease presenting as fever in spinal cord injury. Arch Phys Med Rehabil 68: 176-177.

15 Berlly MH. Wilmot CB (1984) Acute abdominal emergencies during the first four weeks after spinal cord injury. Arch Phys Med Rehabil 65: 687-690.

16 Sugarman B (1984) Osteomyelitis in spinal cord injury. Arch Phys Med Rehabil 65: 132-134.

17 Lal S, Hamilton BB, Heinemann A, Betts HB (1989) Risk factors for heterotopic ossification in spinal cord injury. Arch Phys Med Rehabil 70: 387-390.

18 De Vivo MJ, Kartus PL, Stover SL, Rutt RD, Fine PR (1989) Cause of death for patients with spinal cord injuries. Arch Intern Med 149: 1761-1766.

19 Myllynen P, Kivioga A, Rokkanen P, Wilppula E (1989) Cervical spinal cord injury: the correlations of initial clinical features and blood gas analyses with early prognosis. Paraplegia 27: 19-26.

20 Pembrey MS (1897) The temperature of man and animals after section of the spinal cord. $\mathrm{Br} \mathrm{Med} J$ 2: $883-884$.

21 Sugarman B (1982) Fever in recently injured quadriplegic persons. Arch Phys Med Rehabil 63: 639-640.

22 Atkins E, Bodel P (1972) Fever. N Engl J Med 286: 27-34. 